\section{Synthetische Biologie als Naturwissenschaft mit technischer Ausrichtung} Plädoyer für eine „Hermeneutische Technikfolgenabschätzung"

\section{von Armin Grunwald, ITAS}

Die Synthetische Biologie gehört zu den aktuellen Ausprägungen konvergierender Wissenschaften. Charakteristisch ist ihre Hinwendung zu künstlichen Formen des Lebens - entweder indem existierendes Leben umgestaltet oder (jedenfalls dem Anspruch nach) neues konstruiert wird. Bereits früh haben Technikfolgenabschätzung (TA) und ELSI-Studien zu diesem Forschungsgebiet eingesetzt. Diese können sich noch längst nicht auf ein gut einschätzbares Folgenwissen über Synthetische Biologie stützen. Es stellt sich also die Frage, welche Wissensbestände, Praktiken und Gegenstände in den Blick genommen werden können und welche TA-Schlussfolgerungen und Gestaltungsoptionen überhaupt sinnvoll sind. TA sollte in diesem Feld primär als Hermeneutik gegenwärtiger Visionen und „Erzählungen" über Synthetische Biologie statt als prospektive Folgenforschung operieren.

\section{Einleitung und Zielsetzung}

Die Synthetische Biologie hat sich an neu entstandenen Schnittstellen zwischen der traditionellen Biologie als Naturwissenschaft und den Ingenieurund Technikwissenschaften herausgebildet und ist Folge von technisch ermöglichten Konvergenzen im Wissenschaftssystem (Roco/Bainbridge 2002). Wissenschaftstheoretisch ist Synthetische Biologie eines der immer wieder genannten Paradebeispiele für „Technosciences“, in denen die traditionellen Grenzen zwischen (erkenntnisorientierter) Naturwissenschaft und (anwendungsorientierter) Technikwissenschaft sich auflösen und naturwissenschaftliche Grundlagenforschung ab initio in einen technischen Verwertungszusammenhang gestellt wird. ${ }^{1}$ Wissenschaftliches Handeln und technisches Denken konvergieren in der Synthetischen Biologie - zwar nicht in dem Sinne, dass sie epistemologisch ununterscheidbar werden, aber doch zumindest durch arbeitsteilige Verschränkung und übergeordnete Nutzenerwartung (dazu Teil 2).

Synthetische Biologie ist in ihrem weitaus überwiegenden Teil Laborforschung, in der es um grundlegende Fragen geht, die noch recht weit von konkreten Anwendungen entfernt sind. Zwar reicht die Forschung an einigen Stellen durchaus bis in die Nähe der Praxis. ${ }^{2}$ Die großen Versprechungen einiger Protagonisten der Synthetischen Biologie zur Herstellung künstlicher Organismen zur Produktion von Biomasse oder neuartigen Materialien (Benner/Sismour 2005) sind jedoch Zukunftsmusik, deren Realisierbarkeit und Realisierungszeitraum schwer bis gar nicht einschätzbar ist.

Damit stellt sich, obwohl bereits TA- und ELSI-Studien zur Synthetischen Biologie durchgeführt werden, die Frage nach ihrer Ausrichtung sowie ihren Möglichkeiten und Grenzen. Diese Frage stellt sich für die TA insbesondere deshalb, weil ihre Forschung einerseits handlungs- und entscheidungsrelevant sein soll (und insofern „Impact" erzielen soll ${ }^{3}$, andererseits aber keineswegs klar ist, worauf sich Intervention und Gestaltung in der Synthetischen Biologie überhaupt beziehen können (Teil 3), wenn die direkte Gestaltung biotechnischer Produkte und Systeme kein sinnvolles Ziel sein kann. Stattdessen ist TA als hermeneutische Analyse und Reflexion gefragt, wobei der Gestaltungsraum weniger die Ebene der Technik als vielmehr - neben der aktuellen Forschungspraxis - die der gesellschaftlichen und politischen Debatte zur Synthetischen Biologie ist (Teil 4).

\section{Konvergenz von Biologie und Ingenieur- wissenschaft}

Das traditionelle, naturwissenschaftlich geprägte biologische Selbstverständnis in Richtung auf ein Verstehen von Lebensvorgängen wird in der Synthetischen Biologie (Ball 2005) zu einer Neuerfindung von Natur umgedeutet: Die Schaffung von künstlichem Leben erfolgt auf der Basis des Wissens über das „natürliche“ Leben. Biologie wird dadurch $\mathrm{zu}$ einer technischen Wissenschaft (de Vriend 2006) mit einer Dualität von Erkennen und Gestalten - wie es auch in den klassischen Technikwissenschaften der Fall ist (Banse et al. 2007). Synthetische Biologie ist nach verschiedenen Definitionsvorschlägen (vgl. de Vriend 2006, S. 13ff.): 
-,$\ldots$ the design and construction of new biological parts, devices and systems, and the redesign of existing, natural biological systems for useful purposes“" (LBNL 2006);

-,$\ldots$ the design and synthesis of artificial genes and complete biological systems, and on changing existing organisms, aimed at acquiring useful functions" (COGEM 2006, zit. n. de Vriend 2006, S. 13);

-,$\ldots$ the engineering of biological components and systems that do not exist in nature and the re-engineering of existing biological elements; it is determined on the intentional design of artificial biological systems, rather than on the understanding of natural biology“(Synbiology 2005, S. 1).

Charakteristisch ist allen Definitionen die Hinwendung zu künstlichen Formen des Lebens, die entweder neu konstruiert oder durch Umgestaltung existierenden Lebens erzeugt werden. Voraussetzung für das zielgenaue Design künstlicher Zellen ist ein hinreichendes Verständnis aller erforderlichen subzellulären Prozesse und Wechselwirkungen, wovon der gegenwärtige Wissensstand allerdings noch entfernt ist. Im darauf zielenden Erkenntnisprozess der Synthetischen Biologie geht es darum, Wissen über Strukturen und Funktionen natürlicher Systeme zu erlangen. Lebende Systeme werden in ihrem technischen Funktionszusammenhang analysiert und Zellen als - aus Bauteilen bestehende - Maschinen interpretiert. Beispielsweise werden Proteine und Botenmoleküle als derartige Bauteile begriffen, die in der Synthetischen Biologie nachgebaut, verändert oder neu zusammengesetzt werden können. Dabei wird eine „Modularisierung des Lebens“ vorgenommen und versucht, einzelne Bausteine von Lebensvorgängen zu identifizieren und zu standardisieren. In der Tradition des technischen Standardisierungsdenkens sind im „MIT-Verzeichnis biologischer Standardbauteile" Gensequenzen als Vorlagen für verschiedene Zellmaschinenteile gespeichert. Mit dem gezielten Design von künstlichen Zellen auf der Basis solcher Bauteile sollen Organismen erzeugt werden, indem in der Tradition von Maschinenbau und Elektrotechnik die Bauteile nach einem Bauplan zusammengesetzt werden, um ein funktionsfähiges Ganzes zu erhalten. Entscheidend ist bei diesen Ansätzen die Kombination mo- lekularbiologischen und gentechnischen Wissens mit den Möglichkeiten der Nanotechnologie und der Informationstechnik (Roco/Bainbridge 2002).

Darüber hinaus gibt es Ansätze, Prinzipien der Evolution zu nutzen, um bestimmte neue Effekte zu erreichen. So können z. B. Zellen einem künstlichen Evolutionsdruck ausgesetzt werden, indem bestimmte Gensequenzen ,ausgeschaltet" werden, die für den Aufbau bestimmter Aminosäuren zuständig sind. Durch Zugabe von Substanzen, die der dann fehlenden Aminosäure chemisch hinreichend ähnlich sind, kann die Zelle dazu gebracht werden, für die Biosynthese von Proteinen die Substitute anstelle der originären Aminosäuren zu verwenden. Ergebnis wäre in diesem Fall eine Zelle mit veränderten Eigenschaften. Hier besteht eine enge Schnittstelle zur Systembiologie, in der das Zusammenwirken vieler Einzelvorgänge als ein komplexes Ganzes erfasst werden soll (vgl. die Artikel von Doering und Kollek in diesem Heft).

Im Prozess der Erkennung von Funktionsund Strukturprinzipien der lebenden Natur wird bereits ein technisches Erkenntnisinteresse appliziert (Grunwald/Oertel 2006). Ein Charakteristikum der Synthetischen Biologie besteht konsequenterweise in der Ausweitung der klassischen Ingenieurssprache, v. a. der aus Maschinenbau, Elektrotechnik und Informatik, auf den Bereich des Lebendigen:
„Although it can be argued that synthetic biol- ogy is nothing more than a logical extension of the reductionist approach that dominated biology during the second half of the twentieth century, the use of engineering language, and the practical approach of creating standardised cells and components like in an electrical cir- cuitry suggests a paradigm shift. Biology is no longer considered 'nature at work', but be- comes an engineering discipline." (de Vriend 2006, S. 26).

Beispiele für derartige metaphorisch motivierte Sprachregelungen sind z. B. Hämoglobin als Fahrzeug, Adenosin-Triphosphat-Synthase als Generator, Nukleosome als digitale Datenspeicher, Polymerase als Kopiermaschine oder Membrane als elektrische Zäune (Grunwald/Oertel 2006).

So gesehen und ,versprachlicht" ist Synthetische Biologie erkenntnistheoretisch an eine technische Weltsicht und technische Intervention gebun- 
den. Sie verknüpft den Gedanken des Technischen mit dem Natürlichen, modelliert Natur technomorph und gewinnt aus dieser Perspektive ein bestimmtes Wissen, das dann wieder in die Sphäre des Technischen zurücktransferiert und dort den Ideenvorrat für Problemlösungen erweitern soll. Dabei kommt es - insofern es gelingt, das „Leben“ in technisch reproduzierbare Module zu zerlegen - zu einer erheblichen Erhöhung der Rekombinationsmöglichkeiten und damit zu einer Steigerung der Kontingenz. Mit dem Wachstum des Fundus an Modulen, aus dem sich Technikwissenschaft zur Entwicklung neuer Produkt- und Systemideen bedienen kann, wächst auch die Zahl der Möglichkeiten exponentiell. Und diese Diagnose wiederum stellt ernsthafte Fragen, worum es in einer prospektiven Folgenforschung zur "Gestaltung“ der Synthetischen Biologie überhaupt gehen kann.

\section{Mögliche Gegenstände der Gestaltung}

Nach Verabschiedung technikdeterministischer Vorstellungen, denen es lediglich darum gehen konnte, gesellschaftlich mit den Folgen von Technik umzugehen, geriet die intentionale Gestaltung von Technik nach gesellschaftlichen Werten und Zielen in den Blick. Buchtitel wie ,Shaping Technology - Building Society“ von Bijker and Law drücken den plötzlich sich öffnenden weiten Raum für Gestaltungsoptionen plastisch aus (Bijker/Law 2004). Die Programme des „Social Construction of Technology" (SCOT) (Bijker et al. 1987), der Leitbildforschung (Dierkes et al. 1992) und des Constructive Technology Assessment (CTA, Rip et al. 1995) atmen diesen Geist und haben entsprechenden Optimismus verbreitet. ${ }^{4}$

Ein Stachel im Fleisch dieses Optimismus war stets das „Collingridge-Dilemma“, das sich auf die Gestaltungsmöglichkeiten der Technik selbst, also auf technische Produkte, Anlagen, Systeme oder technikbasierte Dienstleistungen, bezieht. ${ }^{5}$ Das Aufkommen der Technosciences schien die Möglichkeit zu eröffnen, dem Collingridge-Dilemma ein Schnippchen zu schlagen, wurde doch - auch vom Autor dieses Beitrages - die Chance gesehen, bereits in frühen Phasen der Entwicklung durch „upstream engagement“ der TA die Reflexivität der Entwicklung zu erhöhen. Forscher und Entwickler sollten für gesellschaftliche Erwartungen, Werte und Ziele sensibilisiert werden und dazu beizutragen, die technische Entwicklung stärker in Richtung nachhaltige Entwicklung zu beeinflussen. Die Nanotechnologie war hier das Paradebeispiel.

Um diese Erwartungen ist es still geworden. Nanotechnologie wurde ,normalisiert“ (Grunwald/Hocke 2010), ohne dass die hohen Erwartungen an Gestaltung eingelöst werden konnten. Gerade die über zehnjährige Erfahrung in der Gestaltungsdebatte zur Nanotechnologie (Grunwald 2011) gibt Anlass zu einer kritischen Reflexion über berechtigte und weniger berechtigte Erwartungen an Gestaltbarkeit und ihre Gegenstände. Der Slogan „Shaping Technology“ scheint unmittelbar nahezulegen, dass es bei der „Gestaltung“ um die neue Technik selbst in Form von Produkten, Anlagen oder Systemen gehe, die nach gesellschaftlichen Wünschen und Werten gestaltet werden solle - genau wie in der Forderung des CTA nach einer ,,better technology in a better society“ (Rip et al. 1995).

Dieser Eindruck führt jedoch in die Irre. Es handelt sich hierbei um eine Engführung, die die Vielfalt der Gestaltungsmöglichkeiten ausblendet und sich - wie vielfach der frühere Planungsoptimismus - auf die Technik konzentriert und die Möglichkeiten ihrer Gestaltung nach Zielen und Zwecken überschätzt. Dabei wird übersehen, dass es nicht Technik für sich ist, die gestaltet werden muss, sondern komplexe Relationen zwischen Technik und Gesellschaft mit unterschiedlichen Objekten der Gestaltung (Grunwald 2003). Ohne einen Anspruch auf Vollständigkeit zu erheben, können in der weiteren Entwicklung der Synthetischen Biologie folgende Elemente als gestaltbar verstanden werden:

- zukünftige Produkte der Synthetischen Biologie (z. B. könnten bei Designentscheidungen im Labor Prinzipien nachhaltiger Entwicklung angelegt werden),

- zukünftige Wissensbestände der Synthetischen Biologie (dies würde eine aktive Wissenspolitik voraussetzen (Stehr 2004), in der über die positive bzw. negative Wünschbarkeit bestimmter Wissensbestände entschieden wird),

- zukünftigen Forschungsthemen der Synthetischen Biologie (z. B. melden zivilgesellschaftliche Organisationen Mitgestaltungsansprüche an [ETC-Group 2006]; auch die Gestaltung der 
Forschungsförderung würde unter diesen Punkt fallen, ebenso wie die Motivation reflexiver Forschung),

- das Bewusstsein der handelnden Akteure (z. B. durch Sensibilisierung der Biologen für gesellschaftliche Erwartungen und Werte, etwa durch Bildung),

- Akteurskonstellationen (z. B. können durch Netzwerkbildung neue zivilgesellschaftliche Allianzen entstehen),

- rechtlich-politische Rahmenbedingungen (z. B. Regulierung, Anreizsysteme oder Innovationsförderung),

- gesellschaftliche Kommunikation (z. B. durch Medien, aber auch durch Dialoge und Bürgerforen).

Diese Skizze zeigt deutlich, dass Gestaltung im Kontext von Technik keineswegs auf die „Gestaltung von Technik" reduziert werden darf. Letztere Form der Gestaltung ist nur ein Element. Es geht aber darum, komplexe Einbettungs- und Aneignungsprozesse von Technik in die Gesellschaft mit zu gestalten, nicht bloß die Technik als solche. TA dient hierbei als vorbereitende Erforschung und Reflexion solcher verallgemeinerter Gestaltungsprozesse. Was das für eine in frühem Stadium und die Kontingenz zukünftiger Entwicklungen stark erhöhende Forschung wie die Synthetische Biologie bedeutet, sei im Folgenden zumindest kurz angesprochen.

\section{TA als Hermeneutik gegenwärtiger Zukünfte}

Die zunehmende Rekombinierbarkeit der „Module" des Lebens, wie sie von der Synthetischen Biologie erforscht, nachgebaut und modifiziert werden (Teil 2), lässt die Möglichkeit einer Technikgestaltung in der starken Lesart (Grunwald/ Hocke 2010), ja sogar die Möglichkeit einer aussichtsreichen prospektiven Folgenforschung als unrealistisch erscheinen. Jedoch hat sich gezeigt (Teil 3), dass es jenseits der unmittelbaren Gestaltung von Technik als Ergebnis von Forschungsund Entwicklungsprozessen der Synthetischen Biologie ein ganzes Spektrum von Gestaltungsmöglichkeiten anderer Art gibt (,schwache Lesart" nach Grunwald/Hocke 2010). Damit eröffnen sich zwei Typen von Gestaltungsoptionen: (1) die
Gestaltung des gegenwärtigen Forschungsprozesses und (2) die Gestaltung der gegenwärtigen Debatte. Beides bezieht sich, wohlgemerkt, auf die gegenwärtige Situation, nicht auf die Zukunft einer Gesellschaft, in der die Synthetische Biologie mit ihren Produkten Fuß gefasst haben könnte:

(1) Die weitgehende Unmöglichkeit prospektiver Folgenforschung ernst nehmend, kann aussichtsreiche Gestaltung auf den gegenwärtigen Forschungsprozess der Synthetischen Biologie fokussieren. Dieser findet faktisch statt und bedarf keiner prospektiven Analyse, sondern kann z. B. mit den Sorgen der „biosafety“ und der „biosecurity“ konfrontiert werden. Oder es können die Möglichkeiten und Grenzen einer „Doit-yourself"-Technologie betrachtet werden und es kann über die nächsten Forschungsthemen und die Entscheidungsprozesse und -kriterien auf dem Weg dorthin diskutiert werden. Perspektiven und Erfahrungen der TA können hier überall eingehen und den inter- und transdisziplinären Erkenntnisund Gestaltungsprozess bereichern.

(2) Ebenfalls ohne einen Blick in die Zukunft kommt der Ansatz aus, die Visionen und möglicherweise auch anderen „Zukünfte“ der Synthetischen Biologie in den Blick zu nehmen. Denn diese werden ja heute geäußert und bestimmen einen guten Teil der gesellschaftlichen Debatte, deren heilsähnlichen Erwartungen von der Rettung aus der drohenden globalen Energiekrise bis hin zu den Befürchtungen des „Gott-Spielens“ reicht. Gestaltung würde sich hier auf Beiträge zur gesellschaftlichen Debatte erstrecken - mit möglichen, aber nicht zwingenden Folgen für Regulierung. Es würde um die Deutung der Visionen gehen, und um die durch sie ausgelösten Kontroversen. Gestaltung meint hier, die gesellschaftlichen Kontexte der Debatte um Synthetische Biologie so auszugestalten, dass ,verantwortliche Innovation" oder auch ,verantwortliche Forschung und Entwicklung" möglich sind bzw. gefördert werden, wobei diese Konzepte einer inhaltlichen Ausfüllung bedürften (Grunwald 2012). Dabei nimmt die Bedeutung begrifflicher, heuristischer und hermeneutischer Fragen zu. Es ist zu klären, worum es in den betrachteten und oft als spekulativ zu veranschlagenden Entwicklungspotenzialen der Synthetischen Biologie überhaupt geht:

- Was steht auf dem Spiel? 
- Welche Rechte werden möglicherweise beeinträchtigt?

- Welche Menschen-, Natur- und Technikbilder entwickeln sich und wie verändern sie sich?

- Welche anthropologischen Fragen sind involviert und welche Gesellschaftsentwürfe in den Zukunftsprojektionen schwingen mit?

Eine „Hermeneutische TA“ würde einerseits gegenwärtige Debatten über sich selbst aufklären und kommende Debatten vorbereiten, in denen es dann z. B. um die konkrete Technikgestaltung gehen könnte. Ein „Vision Assessment“ (Grunwald 2009) würde in diesem Rahmen technikbasierte Visionen in ihren kognitiven und evaluativen Gehalten und in Bezug auf ihre Folgen untersuchen und wäre grundlegender Baustein eines offenen, kognitiv informierten und normativ orientierten Dialogs - eines Dialogs z. B. zwischen Experten und Öffentlichkeit oder zwischen Synthetischer Biologie, Ethik, Forschungsförderung, Öffentlichkeit und Regulierung.

Für beide Ausprägungen (Selbstaufklärung und Vision Assessment) spielt das CollingridgeDilemma keine Rolle. Es bezieht sich nur auf die direkte Gestaltung der technischen Produkte und Systeme. In der Synthetischen Biologie jedoch geht es um andere Gestaltungsgegenstände und Intentionen in der Erwartung, dass dadurch indirekte Effekte auch auf der Gegenstandsseite der Technik erfolgen. Mit den hier genannten Gestaltungselementen ist jedenfalls nicht die Gefahr verbunden, dass Gestaltung im Sinne eines naiven Kontrolloptimismus fehl interpretiert wird. Auch wenn in einer Hermeneutischen TA sicher die Ziele und Zwecke Synthetischer Biologie auf den Prüfstand gestellt werden, schließt diese Prüfung doch nicht das Auftreten nicht intendierter Nebenfolgen aus. Dieser Erfahrung mit dem technischen Fortschritt verdankt die TA immerhin ihre Motivation und Existenz.

\section{Anmerkungen}

1) Das Konzept der Technosciences setzt eine frühere strikte Trennung zwischen Natur- und Technikwissenschaften voraus. Dies ist jedoch wissenschaftstheoretisch widerlegt, zumindest umstritten (Banse et al. 2007).
2) Beispielsweise wird für das Malaria-Medikament Artemisinin neuerdings ein Ersatzstoff durch Biosynthese in genetisch modifizierten E. coli-Bakterien hergestellt, was immer wieder als eine Annäherung an Synthetische Biologie bezeichnet wird (Dank an PD Dr. Harald König für diese Mitteilung).

3) Siehe dazu Decker/Ladikas 2004.

4) Dieser Optimismus wurde allerdings auch immer wieder als zu voluntaristisch kritisiert (s. z. B. Dolata/Werle 2007).

5) Zum Collingridge-Dilemma siehe die Einleitung zu diesem Schwerpunkt.

\section{Literatur}

Ball, P., 2005: Synthetic Biology for Nanotechnology. In: Nanotechnology 16 (2005), S. R1-R8

Banse, G.; Grunwald, A.; Hronszky, I. et al. (Hg.), 2007: Assessing Societal Implications of Converging Technological Development. Berlin

Benner, S.A.; Sismour, A.M., 2005: Synthetic Biology. In: Nature Reviews/Genetics 6 (2005), S. 533-543

Bijker, W.E.; Hughes, T.P.; Pinch, T.J. (Hg.), 1987: The Social Construction of Technological Systems. Cambridge, MA

Bijker, W.; Law J. (Hg.), 1994: Shaping Technology/ Building Society. Studies in Sociotechnical Change. Cambridge, MA

COGEM - Commissie Genetische Modificatie, 2006: Synthetische biologie. Eenonderzoeksveld met voortschrijdendegevolgen; http://www.cogem. net/showdownload.cfm?objectId=FFFE68BF-151764D9-CC6A0703617AD0C7\&objectType $=$ mark. apps.cogem.contentobjects.publication.download.pdf (download 9.10.12)

de Vriend, H., 2006: Constructing Life. Early Social Reflections on the Emerging Field of Synthetic Biology. The Hague

Decker, M.; Ladikas, M. (Hg.), 2004: Bridges Between Science, Society and Policy. Technology Assessment - Methods and Impacts. Berlin

Dierkes, M.; Hoffmann, U.; Marz, L.,1992: Leitbild und Technik. Zur Entstehung und Steuerung technischer Innovationen. Berlin

Dolata, U.; Werle, R. (Hg.), 2007: Gesellschaft und die Macht der Technik. Sozioökonomischer und institutioneller Wandel durch Technisierung. Frankfurt ETC-Group - Action Group on Erosion, Technology and Concentration, 2006: Open Letter: Global Coalition Sounds the Alarm on Synthetic Biology; http:// www.etcgroup.org (download 9.10.12) 
Grunwald, A., 2003: Die Gestaltung der Technik. In: Grunwald, A. (Hg.): Technikgestaltung zwischen Wunsch und Wirklichkeit. Berlin

Grunwald, A., 2009: Vision Assessment Supporting the Governance of Knowledge - the Case of Futuristic Nanotechnology. In: Bechmann, G.; Gorokhov, V.; Stehr, N. (Hg.): The Social Integration of Science. Institutional and Epistemological Aspects of the Transformation of Knowledge in Modern Society. Berlin, S. 147-170

Grunwald, A., 2011: Ten Years of Research on Nanotechnology and Society - Outcomes and Achievements. In: Zülsdorf, T.B.; Coenen Chr.; Ferrari, A. et al. (Hg.): Quantum Engagements: Social Reflections of Nanoscience and Emerging Technologies. Proceedings der S.Net Konferenz 2010 in Darmstadt. Heidelberg

Grunwald, A., Hocke, P., 2010: The Risk Debate on Nanoparticles: Contribution to a Normalisation of the Science/Society Relationship? In: Kaiser, M.; Kurath, M.; Maasen, S. et al. (Hg.): Governing Future Technologies. Nanotechnology and the Rise of an Assessment Regime. Dordrecht, S. 157-177

Grunwald, A.; Oertel, D., 2006: Potenziale und Anwendungsperspektiven der Bionik. Arbeitsbericht Nr. 108, Büro für Technikfolgen-Abschätzung beim Deutschen Bundestag (TAB), Berlin

LBNL - Lawrence Berkeley National Laboratory, 2006: Homepage; http://www.lbl.gov/ (download 9.10.12)

Rip, A.; Misa, T.; Schot, J. (Hg.), 1995: Managing Technology in Society. London

Rocco, M.C.; Bainbridge, W.S. (Hg.), 2002: Converging Technologies for Improving Human Performance. Arlington

Stehr, N., 2004: The Governance of Knowledge. London

Synbiology, 2005: SYNBIOLOGY. An Analysis of Synthetic Biology Research in Europe and North America; http://www2.spi.pt/synbiology/documents/ SYNBIOLOGY_Literature_And_Statistical_Review. pdf (download 8.11.12)

\section{Kontakt}

Prof. Dr. Armin Grunwald

Institut für Technikfolgenabschätzung und

Systemanalyse (ITAS)

Karlsruher Institut für Technologie (KIT)

Karlstraße 11, 76133 Karlsruhe

Tel.: +49 (0) 721 / 608 - 22500

E-Mail: armin.grunwald@kit.edu

\section{Die Medizin auf dem Weg zur Technowissenschaft?}

Technowissenschaftliche Krankheitsidentitäten und die Schwierigkeit der Technikfolgenabschätzung

von Peter Wehling, Universität Augsburg

Technowissenschaftliche Tendenzen sind seit einiger Zeit auch in der Medizin zu beobachten: Sie resultieren aus einer wissenschaftlichen Dynamik, die zunehmend durch technologische Neuerungen vorangetrieben wird. Die so gewonnenen Erkenntnisse lösen sich vom Erfahrungswissen der Ärzte wie der Patienten und greifen immer direkter und schneller in medizinisch-praktische Handlungskontexte ein. Wie der Beitrag verdeutlicht, stellt die Erzeugung technowissenschaftlicher Identitätsangebote (,Risikoperson“, „Genträger" etc.) ein zentrales Element im Wandel der Medizin zu einer wirkmächtigen Technowissenschaft dar. Die Technikfolgenabschätzung steht hierbei vor neuartigen Problemen, auf die sie unter anderem durch die Initiierung gesellschaftlicher Diskurse über Ziele und Mittel der Medizin zum Erreichen von Gesundheit reagieren sollte.

\section{Einleitung}

Gegenwärtig unterliegt die Medizin sehr vielfältigen und teilweise gegenläufigen Veränderungen, die mit höchst unterschiedlichen Begriffen oder Schlagworten bezeichnet werden: als Ökonomisierung, als Privatisierung, als Verwissenschaftlichung oder auch als Demokratisierung aufgrund der erweiterten Mitsprachemöglichkeiten von Patienten (Löfgren et al. 2011). Ungeachtet solcher Differenzen und der mit ihnen verbundenen Kontroversen dürfte die Feststellung unstrittig sein, dass in der medizinischen Forschung wie auch in der Behandlungspraxis technische Apparaturen und Verfahren eine immer bedeutsamere Rolle spielen. Das Spektrum reicht hier von neuartigen Visualisierungstechniken und Diagnoseverfahren bis hin zu Pflegerobotern und zur sog. Telemedizin. 